\title{
Trends of surface humidity and temperature during 1951-2012 in Beijing, China
}

\author{
QI CHU ${ }^{1}$, ZONGXUE XU ${ }^{1,2}$, DINGZHI PENG ${ }^{1,2}$, XIAOJING YANG ${ }^{1} \&$ \\ GANG YANG ${ }^{1}$ \\ 1 Laboratory of Water and Sediment Sciences, Ministry of Education, College of Water Sciences, Beijing Normal \\ University, Beijing 100875, China \\ 2 Joint Center for Global Change Studies (JCGCS), Beijing 100875, China \\ zongxuexu@vip.sina.com
}

\begin{abstract}
In this paper, two datasets, a long time series (1951-2012) of daily surface observations at one meteorological station and a shorter time series (1979-2012) of three-hourly data with $0.1^{\circ} \times 0.1^{\circ}$ spatial resolution were analysed by using non-parametric methods to identify annual and seasonal variations in surface humidity and temperature. The results reveal that: (1) saturation water vapour pressure increased exponentially with temperature. Actual daily values at Beijing Meteorological Station are very close to the theoretical values estimated by using the simplified Clausius-Clapeyron equation, but with seasonal variations. (2) For both long- and short-term data, clear increasing tendencies of annual saturation specific humidity and temperature are found. Decreasing and drying trends were detected for winter. (3) The annual relative humidity showed a decreasing trend except for some suburban areas, somehow related to the lower temperature and increased specific humidity in those areas. (4) Regional changes in topography and elevation likely influenced trends in surface humidity, while local land use showed little effect on it.
\end{abstract}

Keywords Beijing; specific humidity; relative humidity; trends

\section{INTRODUCTION}

Water vapour is an important natural greenhouse gas related to climate change and global warming (Ding et al., 1987; Dessler, 2008). The variation of water vapour content in the upper troposphere is essential because it has an obvious effect on the balanced budget of radiative forcing. The change of water vapour in the lower atmosphere is also significant, since it determines the geographical distribution and maximum intensity of precipitation, the potential intensity of tropical cyclones and human heat stress (Min et al., 2008).

Surface humidity is the physical element reflecting the characteristics of water vapor in the lower atmosphere, and the commonly used measures are saturation specific humidity, specific humidity and relative humidity, describing the capacity of the atmosphere to hold water, the water content and the degree of moisture, respectively. As in situ observations of surface humidity can be easily influenced by changes of instruments and observation procedures (Dai, 2006), the simplified Clausius-Clapeyron equation (C-C equation) is recommended for practical convenience. Based on the $\mathrm{C}-\mathrm{C}$ equation, the saturation vapour pressure over pure water will increase about $6.577 \mathrm{gkg}^{-1}$, but global-scale and the regional-scale studies indicate that there are slight differences between in situ observations and theoretical values (Dai, 2006; Vincent et al., 2007; Brown et al., 2013). Hence, estimating the parameters of the formula is essential before analysing the trends of surface humidity at local scale.

Beijing, the capital of China, has undergone tremendous changes due to the accelerated development of socio-economics and the rapid expansion of population during the past 50 years. However, negative consequences, such as severe water scarcity, terrible air pollution, serious floods and urban water-logging, all accompany the rapid growth of economics and urbanization. Therefore, accurate quantification of recent changes in air moisture, which is regarded as an indicator of atmospheric quality and extreme precipitation, can be beneficial to clarify the mechanism of climate change and enhance decision-making for sustainable development of water resources and environmental protection in Beijing.

Several studies have investigated the spatial and temporal variability of surface humidity at global and national scales (Wang and Gaffen, 2001; Simmons et al., 2010; Song et al., 2012). Because surface humidity changes exhibit obvious regional characteristics and no higher 
resolution temporal and spatial studies have been made so far in Beijing, this study aims to: (1) analyse the tempo-spatial variability of the annual and seasonal surface humidity based on daily data from one meteorological station and 3-hour gridded datasets covering Beijing area; (2) estimate the parameters of the simplified C-C equation using the long time series of daily in situ observations during 1951-2012, and investigate the accuracy when it is applied in Beijing; (3) qualitatively indicate the local-scale effects on surface humidity, such as topography, elevation and urbanization. Non-parametric methods, such as a five-point triple smoothing method, the Mann-Kendall abrupt detection method and Theil-Sen estimator, are employed in this study. The findings will probably contribute to further understanding of the relationship between air moisture and climate change and reducing uncertainties on air pollution, floods and droughts induced by the variations of surface humidity.

\section{DATA AND METHOD DESCRIPTION}

\section{Data description}

Previous studies mainly analysed the climatology and trends of surface humidity and temperature across China and other regions using observation values at meteorological stations. Especially when the urban effect was investigated, a few urban and rural stations were selected to identify the differences between those areas. However, the actual spatial variations and distributions of surface humidity are poorly known owing to the lack of data. Therefore, in this paper, two datasets: a long time series (1951-2012) of daily in situ observations at Beijing meteorological station, and a shorter time series (1979-2012) of three-hourly data (Kun Yang et al., 2010) with $0.1^{\circ} \times 0.1^{\circ}$ spatial resolution, were used to analyse the variations of surface humidity and temperature. In order to clarify whether urbanization has an effect on the spatial characteristics of surface humidity, urban and rural regions were delineated based on the gridded data.

\section{Method description}

C-C equation The saturation vapour pressure of water is a vital parameter to compute surface humidity. For practical convenience, a simplified formulation called the $\mathrm{C}-\mathrm{C}$ equation is widely used by meteorologists. One of the commonly used empirical equations is as follows:

$$
\operatorname{Es}(\mathrm{T})=6.11 \exp [\mathrm{a}(\mathrm{T}-273.16) /(\mathrm{T}-\mathrm{b})]
$$

where Es denotes the saturation vapour pressure of water $(\mathrm{hPa})$; $\mathrm{T}$ stands for temperature $(\mathrm{K})$; $\mathrm{a}=17.56, \mathrm{~b}=35.86$ over pure ice, and $\mathrm{a}=21.87, \mathrm{~b}=7.66$ over pure water. Saturation specific humidity (Qs) and relative humidity (RH) can be computed by:

$$
\begin{aligned}
& \mathrm{Qs}(\mathrm{T})=0.622 \mathrm{Es}(\mathrm{T}) /(\mathrm{P}-0.378 \mathrm{Es}(\mathrm{T})) \\
& \mathrm{RH}=\mathrm{Q} / \mathrm{Qs}
\end{aligned}
$$

where Qs and Q are saturation specific humidity and humidity $\left(\mathrm{g} \mathrm{kg}^{-1}\right)$, respectively. $\mathrm{P}$ stands for surface air pressure.

Five-point triple smoothing Multi-points smoothing is a simple Low Pass FIR (Finite Impulse Response) filter used to smooth long time series data. It is an effective non-parametric way to make the irregular variations smoother and the trend of the original time series more obvious. The selection of the number of points depends primarily on the length of the sample and the need of practical problems. Five-point triple smoothing, like other secondary smoothing methods, is a commonly used polynomial smoothing method. It reflects the actual trend of sequence variation well, and is particularly suitable for the analysis of trends in relatively short period.

Theil-Sen estimator The Theil-Sen estimator is an unbiased estimator of the true slope in simple linear regression. For many distributions of the response error, this estimator has high asymptotic efficiency relative to least-squares. Estimators with low efficiency require more independent observations to attain the same sample variance of efficient unbiased estimators. 
Besides, it is more robust because it is much less sensitive to outliers: It can tolerate arbitrary corruption of up to $29.3 \%$ of the input data-points without degradation of its accuracy.

\section{RESULTS AND DISCUSSION}

\section{Parameter estimation of C-C equation}

Figure 1 shows the fitting results for the $\mathrm{C}-\mathrm{C}$ equation based on daily observations from Beijing meteorological station during 1951-2012. The solid line denotes the fitting curve of C-C equation calibrated by a nonlinear parameter estimator method using MATLAB, where $a$ is 27.26 , and $b$ is -124.1 , while the dash-dotted line represents the curve using the coefficients over pure water with $a=17.56$ and $b=35.86$. The space between the two dotted lines is the $95 \%$ prediction bound of the fitting curve. The dots represent the mean values in spring (April), summer (June), autumn (November) and winter (January) during this period, respectively.

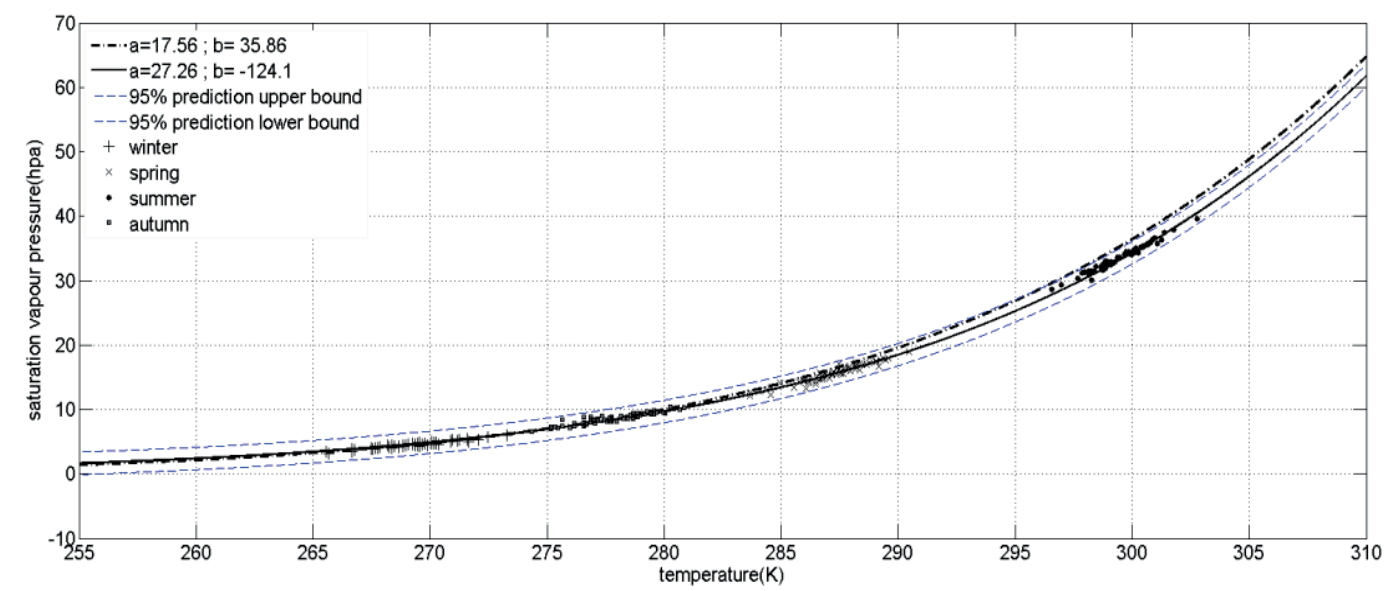

Fig. 1 Linear fitting results of $\mathrm{C}-\mathrm{C}$ equation based on daily in situ observations from Beijing meteorology station during 1951-2012.

It is obvious that saturation vapour pressure increases exponentially with the growth of temperature, which is consistent with the C-C equation. The daily observations agree well with the fitting curve by means of nonlinear parameter estimator method, the correlation coefficient is 0.998. There are obvious differences between the solid line and the dash-dotted line, and this divergence becomes larger with the increase of temperature. The values predicted by the black dash-dotted line are mainly greater than those ones predicted by the solid line, means that the values calculated by the coefficients over pure water overestimate the actual surface water vapour pressure, especially in the urban area, which may be widely influenced by the local climate change and human impact. When the temperature exceeds $295 \mathrm{~K}$, the black dash-dotted line is out of the $95 \%$ confidence boundary of the solid line, indicating that estimating the coefficients is essential before analysing the trends of surface humidity at local scale.

According to the seasonal observations, the magnitude of saturation vapour pressure presents the characteristics of summer $>$ spring $>$ autumn $>$ winter. Also, the observations fit best in winter and worst in spring, which is somehow related to the large diurnal temperature changes and windy climatology in spring.

\section{Trends and variations}

(1) Temporal annual trends during 1951-2012 Figure 2 shows the temporal variations of annual surface humidity and temperature based on daily observations during 1951-2012. The bars represent the time series of annual surface humidity and temperature anomaly averaged. The solid line is the five-point triple smooth curve and the dotted line indicates the linear trend using the Theil-Sen estimator. 
As shown in Fig. 2, annual T and Qs exhibit an obvious upward trend while Q and RH show a reducing tendency, concurring with the findings for urban areas provided by Zheng (2008). The increase rate of $\mathrm{T}$ is $0.4-0.5^{\circ} \mathrm{C} / 10$-years, which is a little higher than the global mean. The trends of Qs are consistent with the growth of T, showing an upward rate of $0.002-0.004 \mathrm{~g} \mathrm{~kg}^{-1} / 10$-years. Partly due to the influence of local climate change and human activities, the inter-annual variations of $\mathrm{Q}$ are more random and larger than $\mathrm{Q}$, which leads to a sharp reducing tendency of RH.

Through the five-point triple smoothing method, an obvious opposite tendency was found for T, Qs and RH before and after 1980, which is the turning point of these elements detected by using the Mann-Kendall method. Ding (2006) concluded that China has undergone two obvious warming trends, during the 1930s (1920-1940) and 1990s (1980-2006), and the recent warming since 1980 is correlated with the sharp rise of greenhouse gases. For both $\mathrm{T}$ and Qs, the annual anomalies appear negative before the 1980s while positive after the 1980s. Unlike T and Qs, the annual RH anomalies seem to decrease in the first 10 years and then exhibit a significant upward trend during 1960-1980. After 1980, a dramatic reduction is found, which may be one of the important reasons why decreasing rainfall occurred in recent years.

Beijing Meteorological Station is located in the southern urban area of Beijing. It can be concluded that this local area presents a warm-dry tendency after the 1980 s, partly correlated with the increasing capacity of the atmosphere to hold water and small changes in evaporation, due to slight changes in local climate conditions and land use.
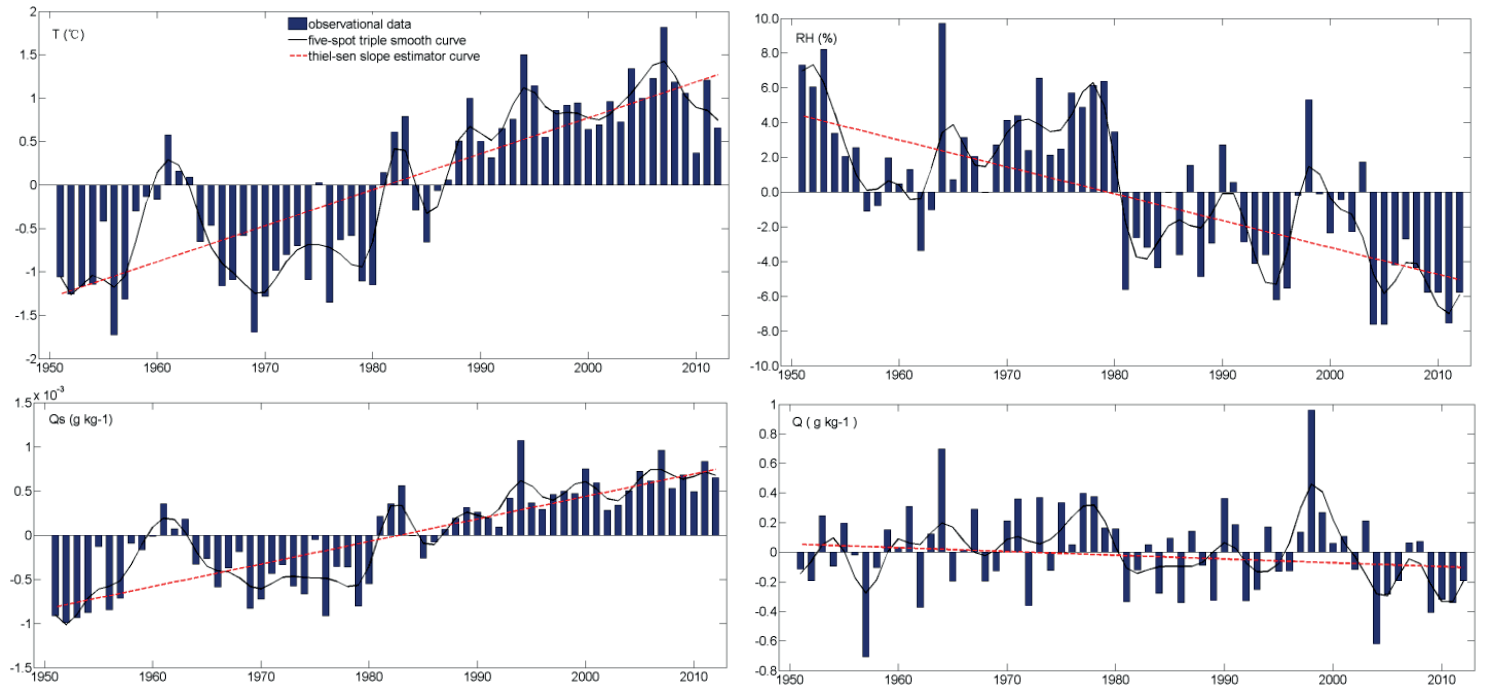

Fig. 2 Trends of surface humidity and temperature based on daily observations from Beijing Meteorological Station during 1951-2012.

(2) Climatology and spatial annual trends during 1979-2012 Figure 3 displays the spatial distribution map of annual median values of surface humidity and temperature based on the 3-h gridded data during the period of 1979-2012. The range of annual temperature was mainly from 1.5 to $16.0^{\circ}$, with the tendency of increase from northwest to southeast, somehow correlated with the distribution of elevation and topography. Saturation specific humidity shows similar spatial features, with values between 5.0 and $9.0 \mathrm{~g} \mathrm{~kg}^{-1}$, which is $1.5 \mathrm{~g} \mathrm{~kg}^{-1}$ higher than specific humidity. It is worthy to note that, compared with the spatial distribution of $\mathrm{T}$ and $\mathrm{Qs}, \mathrm{Q}$ has relatively less relationship with elevation and topography, especially in the southern area. Due to different upward magnitudes of Qs and Q, RH decreased from northeast to southwest with values of $71 \%-81 \%$.

Figure 4 presents the trends of annual surface humidity and temperature based on the threehourly gridded data using the Theil-Sen slope estimator. According to Fig. 3, for both datasets, clear increasing trends of annual saturation specific humidity and temperature are found. Furthermore, the variations of the area where Beijing meteorological station is located are similar 

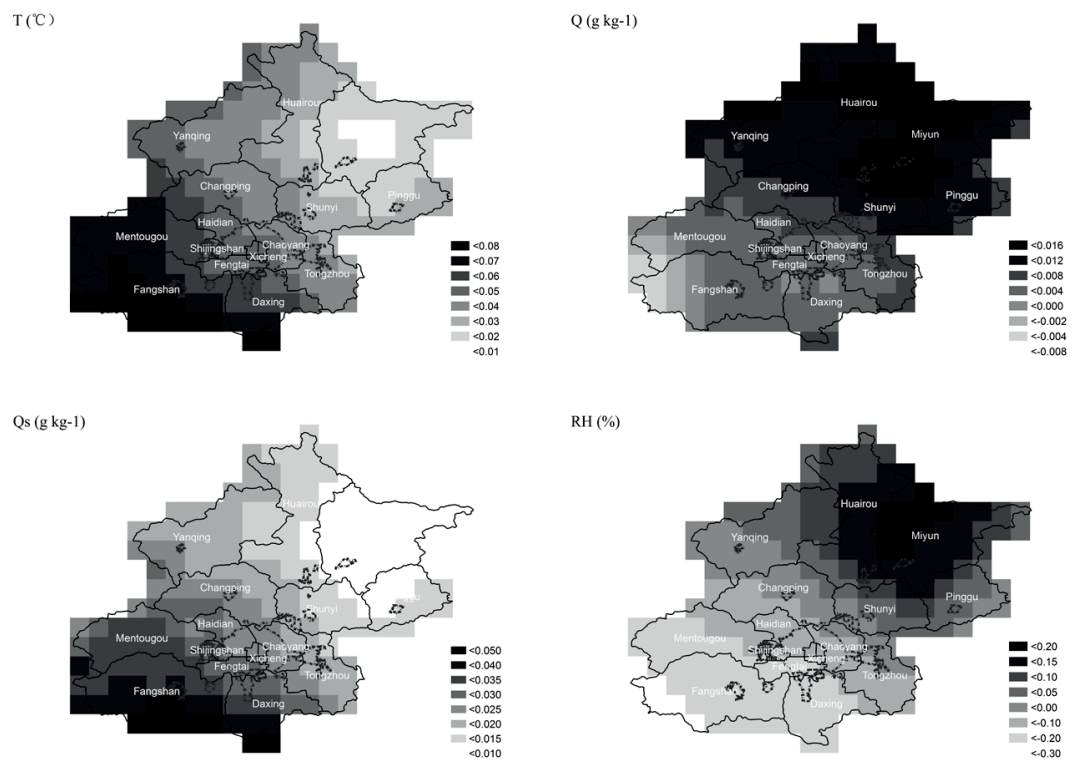

Fig. 3 Climatological annual-mean values of surface humidity and temperature based on 3-hour gridded data during 1979-2012.
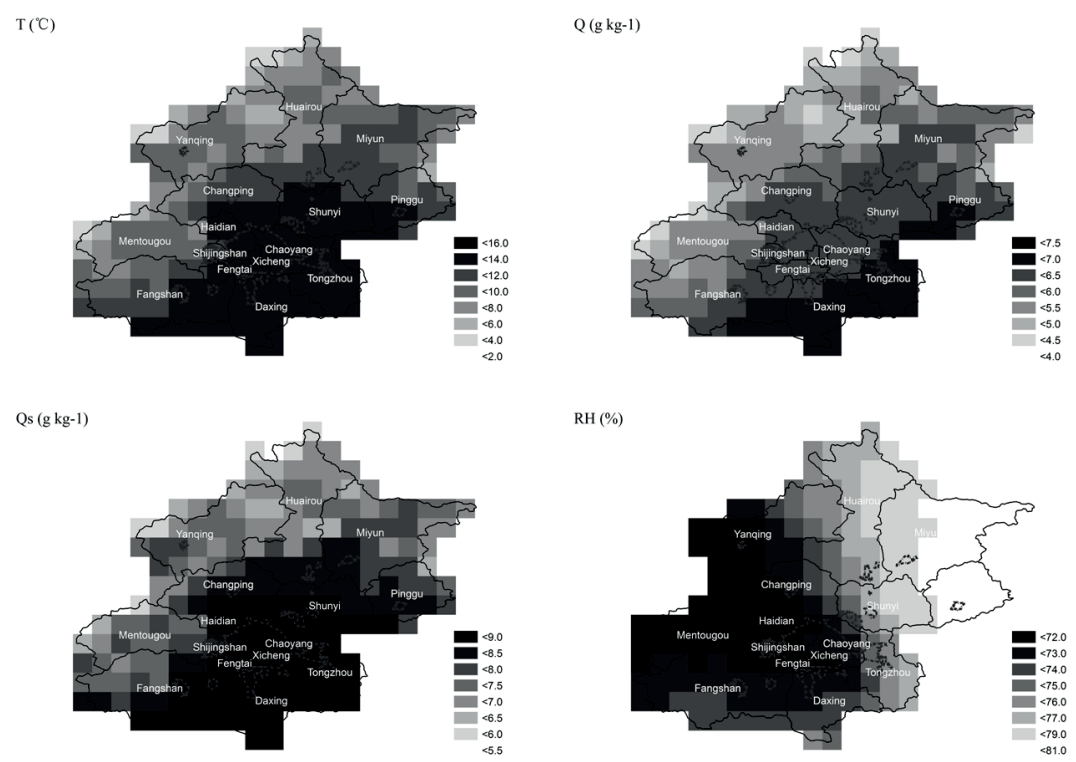

Fig. 4 Trends of surface humidity and temperature based on 3-hour gridded data during 1979-2012.

to the results derived from the in situ observations since 1979. For winter, the decreasing and drying trends are detected, with the tendency from northeast to southwest. Specific humidity also shows an increasing trend, but with the contrary spatial distribution. Except for part of the northeast regions, such as Miyun County, the increasing magnitude of specific humidity is smaller than saturation specific humidity, leading to simultaneous existence of upward and downward trends of RH in Beijing.

The region surrounded by the blue solid line represents the urban area around 2000. The area is mainly in southern Beijing, where annual median temperature is $14^{\circ} \mathrm{C}$, saturation specific humidity is $9.0 \mathrm{~g} \mathrm{~kg}^{-1}$, specific humidity is $7.0 \mathrm{~g} \mathrm{~kg}^{-1}$ and relative humidity is $72 \%$. According to the climatology and trends map of surface humidity and temperature, it can be seen that the 
southern part of Beijing, including the urban areas, indicates a warm-dry trend while the northeastern area becomes warmer and wetter. The spatial differences of sustained intensified relative humidity, indirectly reflect the rising possibility of rainstorm and drought.

\section{CONCLUSIONS}

(1) The saturation vapour pressure increases exponentially with the growth of temperature, which is consistent with the simplified Clausius-Clapeyron equation. The annual observations from Beijing Meteorological Station are very close to the theoretical values, but with seasonal variations. When the temperature is greater than $295 \mathrm{~K}$, the curve which directly used the coefficients over the pure water is out of $95 \%$ confident boundary. Therefore, it is advised to estimate these parameters before analysing the trends of surface humidity in local areas.

(2) The trends of surface humidity and temperature showed opposite tendencies before 1980 and after 1980, in accordance with the climate variations that happened around 1980. For both long- and short-term data, clear increasing trends of annual saturation specific humidity and temperature are found. While for winter, the decreasing and drying trends are detected, with the tendency from northeast to southwest. Specific humidity also showed an increasing trend, but with the contrary spatial distribution. Except for parts of the northeast regions, such as Miyun County, the increased magnitude of specific humidity is smaller than the saturation specific humidity, leading to simultaneous upward and downward trends of RH in Beijing.

(3) According to the spatial distribution and the trends of surface humidity, it can be seen that the southern part of Beijing presents a warm-dry trend while the northeastern area becomes warmer and wetter. The spatial differences of relative humidity are sustained and intensified, indicating the rising possibility of rainstorm and drought.

(4) The tendency of urban-related warming and drying is observed, which is strongest in winter. Regional changes in topography and elevation likely affect the trends in surface humidity, while local land use has little effect on it.

Acknowledgements This study was supported by the research project from Beijing Natural Science Foundation (No. 8141003). The authors thank the Data Assimilation and Modelling Centre for Tibetan Multi-spheres (DAM) for providing high resolution dataset of surface humidity and temperature.

\section{REFERENCES}

Brown P.J. and Degaetano, A.T. (2013) Trends in U.S. surface humidity, 1930-2010. Journal of Applied Meteorology and Climatology 02, 147-163.

Dai, A.G. (2006) Recent climatology, variability, and trends in global surface humidity. Journal of Climate 19, 3589-3606.

Dessler, A.W., Zhang, Z. and Yang, P. (2008) Water vapor climate feedback inferred from climate fluctuation. Geophysical Resources Letter, 35:L20704.

Ding Y.H. et al. (2006) National Assessment Report of Climate Change: Climate change in China and its future trend. Advances in Climate Change Research 02, 3-8.

Ding, Y.H. and Krishnamuriti, T.N. (1987) Heat budget of the Siberian high and the winter monsoon. Mon. Weather Rev. 115, 2428-2449.

Kun Yang, et al. (2010) On downward shortwave and longwave radiations over high altitude regions: Observation and modeling in the Tibetan Plateau. Agriculture Forest Meteorology 150, 38-46.

Min, S.K., Zhang, X. and Williams Jr., C.N. (2008) Human induced Arctic moistening. Science 320, 518-520.

Murray, F.W. (1967)On the computation of saturation vapour pressure. Journal of Applied Meteorology 6, 203-204.

Song, Y.F., Liu, Y.J. and Ding, Y.H. (2012)A study of surface humidity changes in China during the recent 50 years. Acta Meteorologica Sinica 26, 541-553.

Vincent, L.A. Van Wijngaarden, W.A. and Hopkinson, R. (2007) Surface temperature and humidity trends in Canada for 19532005. Journal of Climate 20, 5100-5113.

Wang, J.X.L. and Gaffen, D.J. (2000) Late-twentieth-century climatology and trends of surface humidity and temperature in China. Journal of Climate 14, 2833-2845.

Zheng, S.Y. and Liu, S.H. (2008) Urbanization effect on climate in Beijing. Climate and Environmental Research 13, $123-133$. 